\author{
N. T. Orumbayeva ${ }^{1,2}$, G. Sabitbekova ${ }^{3}$ \\ ${ }^{1}$ Institute of Applied Mathematics, Karaganda, Kazakhstan; \\ ${ }^{2}$ Ye.A.Buketov Karaganda State University, Kazakhstan; \\ ${ }^{3}$ I.Altynsarin Arkalyk State Pedagogical Institute, Kazakhstan \\ (E-mail: OrumbayevaN@mail.ru)
}

\title{
A boundary value problem for nonlinear differential equation with arbitrary functions
}

\begin{abstract}
This article describes a semi-batch nonlinear boundary value problem for differential equations with partial derivatives. The equations containing arbitrary parameters were considered in Whitham G.B. Such equations are encountered in some problems of chemical technology and chromatography. Replacing $u=e^{k z}$ in a nonlinear problem with arbitrary functions leads to a semi-batch linear boundary value problem for hyperbolic equations. Introducing a new unknown function, semi-batch linear boundary value problem for hyperbolic equations with mixed derivative reduced to the family of boundary value problems for ordinary differential equations and functional relation. Using the method of parameterization to the family of boundary value problems for ordinary differential equations, We find approximate solutions of equations in this area. The proposed method is illustrated by an example.
\end{abstract}

Keywords: nonlinear equation, boundary value problem, differential equation in partial derivatives, hyperbolic equation.

On $\Omega=[0, X] \times[0, Y]$ is considered for periodic boundary value problem nonlinear differential equations with partial derivatives

$$
\begin{gathered}
\frac{\partial^{2} z}{\partial x \partial y}=k \cdot \frac{\partial z}{\partial x} \cdot \frac{\partial z}{\partial y}+a(x, y) \cdot \frac{\partial z}{\partial x}+f(x, y) \\
z(0, y)=\psi(y) \\
z(x, 0)=z(x, T)
\end{gathered}
$$

where $k=$ const, $\varphi(y)$ - given function depending on $y, a(x, y), f(x, y)$ - arbitrary functions depending on $x$ and $y$. The paper G.B.Whitham [1] were considered equations containing arbitrary parameters. Such equations are encountered in some problems of chemical technology and chromatography.

To solve the problem (1)-(3) $u=e^{k z}$ we make the change, then we obtain a linear periodic boundary value problem

$$
\begin{gathered}
\frac{\partial^{2} u}{\partial x \partial y}=a(x, y) \cdot \frac{\partial u}{\partial y}+k \cdot f(x, y) \cdot u \\
u(0, y)=e^{k \psi(y)} ; \\
u(x, 0)=z(x, Y) ; \\
z(x, y)=\frac{1}{k} \ln u(x, y) .
\end{gathered}
$$

We make a partition by $\tau>0, h>0: M \tau=X, N h=Y$ step

$$
[0, X)=\bigcup_{i=1}^{M}[(i-1) \tau, i \tau), \quad[0, Y)=\bigcup_{j=1}^{N}[(j-1) h, j h), \quad M \geq 2, \quad N \geq 2 .
$$

In this region $\Omega$ is divided into $M \times N$ parts. By $u_{i j}(x, t), z_{i j}(x, t)$ denote the restriction of $u(x, t), z(x, t)$ in area $\Omega_{i j}=[(i-1) \tau, i \tau) \times[(j-1) h, j h), \quad i=\overline{1, M}, \quad j=\overline{1, N}[2]$.

To find a solution we insert a new unknown function $v_{i j}(x, t)=\frac{\partial u_{i j}(x, t)}{\partial x}, i=\overline{1, M}, j=\overline{1, N}$, we insert the notation $\lambda_{i j}(x)=v_{i j}(x,(j-1) h)$ and make the change $\widetilde{v}_{i j}(x, t)=v_{i j}(x, t)-\lambda_{i j}(x), \quad i=\overline{1, M}, j=\overline{1, N}$. We obtain the equivalent boundary value problem with unknown functions $\lambda_{i j}(x)$ :

$$
\frac{\partial \widetilde{v}_{i j}}{\partial y}=a(x, y) \widetilde{v}_{i j}+a(x, y) \lambda_{i j}(x)+k f(x, y) u_{i j}(x, y), \quad(x, t) \in \Omega_{i j}, \quad i=\overline{1, M}, \quad j=\overline{1, N}
$$




$$
\begin{gathered}
\widetilde{v}_{i j}(x,(r-1) h)=0 ; \\
\lambda_{i 1}(x)-\lambda_{i N}(x)-\lim _{y \rightarrow Y-0} \widetilde{v}_{i N}(x, y)=0 ; \\
\lambda_{i s}(x)+\lim _{y \rightarrow s h-0} \widetilde{v}_{i s}(x, y)-\lambda_{i, s+1}(x)=0, \quad s=\overline{1, N-1} ; \\
u_{i j}(x, y)=e^{k \varphi(y)}+\int_{0}^{x}\left[\widetilde{v}_{i j}(\xi, y)+\lambda_{i j}(\xi)\right] d \xi ; \\
z_{i j}(x, y)=\frac{1}{k} \ln u_{i j}(x, y) .
\end{gathered}
$$

Task (8), (9) with fixed $\lambda_{i j}(x), u_{i j}(x, y)$ is the solution of the Cauchy problem and is equivalent to the equation

$$
\widetilde{v}_{i j}(x, y)=\int_{(j-1) h}^{y} a(x, \eta) \widetilde{v}_{i j}(x, \eta) d \eta+\int_{(j-1) h}^{y} a(x, \eta) d \eta \cdot \lambda_{i j}(x)+\int_{(j-1) h}^{y} k f(x, \eta) u_{i j}(x, \eta) d \eta .
$$

Passing on the right (14) to the limit $y \rightarrow Y-0, \quad y \rightarrow s h-0$ substituting them into equation (10), (11) for unknown functions $\lambda_{i j}(x)$, we obtain a system of functional equations:

$$
\begin{gathered}
\lambda_{i 1}(x)-\lambda_{i N}(x)-\lambda_{i N}(x) \int_{(N-1) h}^{Y} a(x, \eta) d \eta=\int_{(N-1) h}^{Y} a(x, \eta) \widetilde{v}_{i N}(x, \eta) d \eta+k \int_{(N-1) h}^{Y} f(x, \eta) u_{i j}(x, \eta) d \eta ; \\
\lambda_{i s}(x)+\lambda_{i s}(x) \int_{(s-1) h}^{s h} a(x, \eta) d \eta-\lambda_{i, s+1}(x)= \\
=-\int_{(s-1) h}^{s h} a(x, \eta) \widetilde{v}_{i s}(x, \eta) d \eta-k \int_{(s-1) h}^{s h} f(x, \eta) u_{i s}(x, \eta) d \eta, \quad s=\overline{1, N-1} .
\end{gathered}
$$

To find the system of three functions $\left\{\lambda_{i j}(x), \widetilde{v}_{i j}(x, y), u_{i j}(x, y), z_{i j}(x, y)\right\}, \quad i=\overline{1, M}, \quad j=\overline{1, N}$, we have a closed system comprised of equations (15), (16), (14),(12),(13).

For an initial approximation of the problem (8)-(9) we take $\widetilde{v}_{1 j}^{(0)}(x, t)=0, u_{1 j}^{(0)}(x, t)=e^{k \psi(y)}, j=\overline{1, N}$, and successive approximations based on the following algorithm:

Step 1. For $\widetilde{v}_{1 j}^{(0)}(x, t)=0, \quad u_{1 j}^{(0)}(x, t)=e^{k \psi(y)}$ from the equations (15) and (16) we find the $\lambda_{1 j}^{*}(x), j=\overline{1, N}$. Using (14) and found $\lambda_{1 j}^{*}(x)$, define $\widetilde{v}_{1 j}^{*}(x, t)$. Then, from the equation (12) get $u_{1 j}^{*}(x, y)$. Next, using (13) find $z_{2 j}^{*}(x, y)$.

Step 2. For $\widetilde{v}_{2 j}^{(0)}(x, t)=0, u_{2 j}^{(0)}(x, t)=\lim _{x \rightarrow \tau-0} u_{1 j}^{*}(x, t)$ from the equation (15) and (16) get $\lambda_{2 j}^{*}(x), j=\overline{1, N}$. Using (14) and found $\lambda_{2 j}^{*}(x)$, define $\widetilde{v}_{2 j}^{*}(x, t)$. Then, from the equation (12), (13) get $u_{2 j}^{*}(x, t), z_{2 j}^{*}(x, t), j=\overline{1, N}$.

Step M. For $\widetilde{v}_{M j}^{(0)}(x, t)=0, u_{M j}^{(0)}(x, t)=\lim _{x \rightarrow(M-1) \tau-0} u_{M-1, j}^{*}(x, t)$ from the equations (15) and (16) we find the $\lambda_{M j}^{*}(x), j=\overline{1, N}$. Using (14) and found $\lambda_{M j}^{*}(x)$, define $\widetilde{v}_{M j}^{*}(x, t)$. Then, from the equation (12), (13) get $u_{M j}^{*}(x, t), z_{M j}^{*}(x, t), j=\overline{1, N}$.

Example. On the $\Omega=[0,1] \times[0,1]$ is considered the boundary value problem $k=1, a(x, y)=-2 x y$.

$$
\begin{gathered}
\frac{\partial^{2} z}{\partial x \partial y}=\frac{\partial z}{\partial x} \cdot \frac{\partial z}{\partial y}-2 x y \cdot \frac{\partial z}{\partial x}+2 y-1+x y^{2}-x y \\
z(0, y)=0 \\
z(x, 0)=z(x, 1) .
\end{gathered}
$$


To solve the problem (17)-(19) $u=e^{z}$ we make the change, then we obtain a linear periodic boundary value problem

$$
\begin{gathered}
\frac{\partial^{2} u}{\partial x \partial y}=-2 x y \cdot \frac{\partial u}{\partial y}+\left(2 y-1+x y^{2}-x y\right) \cdot u \\
u(0, y)=1 ; \\
u(x, 0)=u(x, 1) \\
z(x, y)=\ln u(x, y) .
\end{gathered}
$$

Let $\tau=\frac{1}{2}, h=\frac{1}{2}, N=2$. To find a solution we insert a new unknown function $v_{i j}(x, t)=\frac{\partial u_{i j}(x, t)}{\partial x}$, $i, j=1,2$, we insert the notation $\lambda_{i j}(x)=v_{i j}(x,(j-1) h), \quad \Omega_{i j}=\left[\frac{i-1}{2}, \frac{i}{2}\right) \times\left[\frac{j-1}{2}, \frac{j}{2}\right)$ and make the change $\widetilde{v}_{i j}(x, t)=v_{i j}(x, t)-\lambda_{i j}(x), i, j=1,2$. We obtain the equivalent boundary value problem with unknown functions $\lambda_{i j}(x)$ :

$$
\begin{gathered}
\frac{\partial \widetilde{v}_{i j}}{\partial y}=-2 x y \widetilde{v}_{i j}-2 x y \lambda_{i j}(x)+\left(2 y-1+x y^{2}-x y\right) u_{i j}(x, y), \quad \widetilde{v}_{i j}(x, 0)=0, \quad(x, t) \in \Omega_{i j}, i, j=1,2 ; \\
\lambda_{i 1}(x)-\lambda_{i 2}(x)-\lim _{y \rightarrow 1-0} \widetilde{v}_{i 2}(x, y)=0 ; \\
\lambda_{i 1}(x)+\lim _{y \rightarrow \frac{1}{2}-0} \widetilde{v}_{i 1}(x, y)-\lambda_{i 2}(x)=0 ; \\
u_{1 j}(x, y)=1+\int_{0}^{x}\left[\widetilde{v}_{1 j}(\xi, y)+\lambda_{1 j}(\xi)\right] d \xi ; \\
u_{2 j}(x, y)=\lim _{x \rightarrow \frac{1}{2}-0} u_{1 j}(x, y)+\int_{\frac{1}{2}}^{x}\left[\widetilde{v}_{2 j}(\xi, y)+\lambda_{2 j}(\xi)\right] d \xi ; \\
z_{i j}(x, y)=\ln u_{i j}(x, y) .
\end{gathered}
$$

Task (24) with fixed $\lambda_{i j}(x), u_{i j}(x, y)$ is the solution of the Cauchy problem and is equivalent to the equation

$$
\widetilde{v}_{i j}(x, y)=-2 \int_{\frac{j-1}{2}}^{y} x \eta \widetilde{v}_{i j}(x, \eta) d \eta-2 \int_{\frac{j-1}{2}}^{y} x \eta d \eta \cdot \lambda_{i j}(x)+\int_{\frac{j-1}{2}}^{y}\left(2 \eta-1+x \eta^{2}-x \eta\right) u_{i j}(x, \eta) d \eta, \quad i, j=1,2 .
$$

Passing on the right (30) to the limit $\lim _{y \rightarrow 1-0} \widetilde{v}_{i 2}(x, y), \lim _{y \rightarrow \frac{1}{2}-0} \widetilde{v}_{i 1}(x, y), i=1,2$, substituting them into equation (25), (26), for unknown functions $\lambda_{i j}(x), i, j=1,2$, we obtain a system of functional equations:

$$
\begin{aligned}
& \lambda_{i 1}(x)-\lambda_{i 2}(x)+2 \int_{\frac{1}{2}}^{1} x \eta \widetilde{v}_{i 2}(x, \eta) d \eta+2 \int_{\frac{1}{2}}^{1} x \eta d \eta \cdot \lambda_{i 2}(x)-\int_{\frac{1}{2}}^{1}\left(2 \eta-1+x \eta^{2}-x \eta\right) u_{i 2}(x, \eta) d \eta=0 \\
& \lambda_{i 1}(x)-2 \int_{0}^{\frac{1}{2}} x \eta \widetilde{v}_{i 1}(x, \eta) d \eta-2 \int_{0}^{\frac{1}{2}} x \eta d \eta \cdot \lambda_{i 1}(x)+\int_{0}^{\frac{1}{2}}\left(2 \eta-1+x \eta^{2}-x \eta\right) u_{i 1}(x, \eta) d \eta-\lambda_{i 2}(x)=0 .
\end{aligned}
$$

For an initial approximation of the problem $(24)-(29)$ we take $\widetilde{v}_{1 j}^{(0)}(x, y)=0, u_{1 j}^{(0)}(x, y)=1, j=1,2$, and successive approximations based on the following algorithm:

Step 1. For $\widetilde{v}_{1 j}^{(0)}(x, y)=0, u_{1 j}^{(0)}(x, y)=1, j=1,2$, from the equations (31) and (32) we find the $\lambda_{11}^{*}(x)$, $\lambda_{12}^{*}(x)$. Then

$$
\lambda_{11}(x)=\frac{12+4 x-6 x^{2}}{48-12 x+9 x^{2}}, \quad \lambda_{12}(x)=\frac{11 x-x^{2}}{48-12 x+9 x^{2}} ;
$$




$$
\max _{x \in\left[0, \frac{1}{2}\right)} \lambda_{11}(x)=0.2834, \quad \max _{x \in\left[0, \frac{1}{2}\right)} \lambda_{12}(x)=0.1186
$$

Using (30) and results $\lambda_{11}^{*}(x), \lambda_{12}^{*}(x)$, define $\widetilde{v}_{11}^{*}(x, y), \widetilde{v}_{12}^{*}(x, y)$ :

$$
\begin{gathered}
\widetilde{v}_{11}^{*}(x, y)=-2 \cdot \lambda_{11}^{*}(x) \int_{0}^{y} x \eta d \eta+\int_{0}^{y}\left(2 \eta-1+x \eta^{2}-x \eta\right) d \eta= \\
=0.3333 y\left(x y^{2}+(3-2.3502 x) y-3\right) ; \\
\widetilde{v}_{12}^{*}(x, y)=-2 \cdot \lambda_{12}(x) \int_{\frac{1}{2}}^{y} x \eta d \eta+\int_{\frac{1}{2}}^{y}\left(2 \eta-1+x \eta^{2}-x \eta\right) d \eta= \\
=0.3333 x y^{3}-0.6186 x y^{2}+0.1130 x+y^{2}-y+0.25 ; \\
\max _{x \in\left[0, \frac{1}{2}\right)} \widetilde{v}_{11}^{*}(x, y)=y^{2}-y, \quad \max _{x \in\left[0, \frac{1}{2}\right)} \widetilde{v}_{12}^{*}(x, y)=y^{2}-y+0.25 .
\end{gathered}
$$

Further, from the equations $(27)$ get $u_{11}^{*}(x, y), u_{12}^{*}(x, y)$ :

$$
u_{11}^{*}(x, y)=1+x y^{2}-x y+0.2834 x, \quad u_{12}^{*}(x, y)=1+x y^{2}-x y+0.3686 x .
$$

Next, using (29) find $z_{11}^{*}(x, y), z_{12}^{*}(x, y)$.

Step 2. For $\widetilde{v}_{21}^{(0)}(x, y)=0, \widetilde{v}_{22}^{(0)}(x, y)=0$,

$$
\begin{aligned}
& u_{21}^{(0)}(x, y)=\lim _{x \rightarrow \frac{1}{2}-0} u_{11}^{*}(x, y)=0.5 y^{2}-0.5 y+1.1417 \\
& u_{22}^{(0)}(x, y)=\lim _{x \rightarrow \frac{1}{2}-0} u_{12}^{*}(x, y)=0.5 y^{2}-0.5 y+1.1843
\end{aligned}
$$

from the equations (31) and (32) we find the $\lambda_{21}^{*}(x), \lambda_{22}^{*}(x)$.

Then

$$
\begin{gathered}
\lambda_{21}^{*}(x)=\frac{0.3708\left(x^{2}+0.4146 x-0.069\right)}{5.3333-x^{2}}, \quad \lambda_{22}^{*}(x)=\frac{0.1195\left(x^{2}-11.2266 x-0.2143\right)}{5.3333-x^{2}} ; \\
\max _{x \in\left[\frac{1}{2}, 1\right)} \lambda_{21}(x)=0.1151, \quad \max _{x \in\left[\frac{1}{2}, 1\right)} \lambda_{22}(x)=-0.1405 .
\end{gathered}
$$

Using (30) and results $\lambda_{21}^{*}(x), \lambda_{22}^{*}(x)$, define $\widetilde{v}_{21}^{*}(x, y), \widetilde{v}_{22}^{*}(x, y)$ :

$$
\begin{gathered}
\widetilde{v}_{21}^{*}(x, y)=y\left(y^{3}(0.25-0.25 x)+y(1.3917-0.6859 x)+0.1 x y^{4}+(0.5472 x-0.5) y^{2}-1.1417\right) ; \\
\widetilde{v}_{22}^{*}(x, y)=0.1 x y^{5}-0.25 x y^{4}+0.5614 x y^{3}-0.4516 x y^{2}+0.0552 x+ \\
+0.25 y^{4}-0.5 y^{3}+1.4343 y^{2}-1.1843 y+0.2804 ; \\
\max _{x \in\left[\frac{1}{2}, 1\right)} \widetilde{v}_{21}^{*}(x, y)=0.1 y^{5}+0.0472 y^{3}+0.7058 y^{2}-1.1417 y ; \\
\max _{x \in\left[\frac{1}{2}, 1\right)} \widetilde{v}_{22}^{*}(x, y)=0.1 y^{5}+0.0614 y^{3}+0.9827 y^{2}-1.1843 y+0.3356 .
\end{gathered}
$$

Further, from the equation (28) get $u_{21}^{*}(x, y), u_{22}^{*}(x, y)$. Thus, to solve this problem

$$
\begin{array}{ll}
z_{11}^{*}(x, y)=\ln \left(1+x y^{2}-x y+0.2834 x\right), & x \in\left[0, \frac{1}{2}\right), \quad y \in\left[0, \frac{1}{2}\right) \\
z_{12}^{*}(x, y)=\ln \left(1+x y^{2}-x y+0.3686 x\right), & x \in\left[0, \frac{1}{2}\right), \quad y \in\left[\frac{1}{2}, 1\right)
\end{array}
$$




$$
\begin{gathered}
z_{21}^{*}(x, y)=\ln \left(0.1471 y^{2}+0.0708 y+1.0842+0.1 x y^{5}+0.0472 x y^{3}+\right. \\
\left.+0.7058 x y^{2}-1.1417 x y+0.1151 x\right), \quad x \in\left[\frac{1}{2}, 1\right), y \in\left[0, \frac{1}{2}\right) ; \\
z_{22}^{*}(x, y)=\ln \left(1.0165+0.3356 x+0.0126 y-1.1843 x y+0.0086 y^{2}+\right. \\
\left.+0.9827 x y^{2}-0.0307 y^{3}+0.0614 x y^{3}-0.05 y^{5}+0.1 x y^{5}\right), \quad x \in\left[\frac{1}{2}, 1\right), \quad y \in\left[\frac{1}{2}, 1\right) .
\end{gathered}
$$

Thus, a nonlinear semi-periodic boundary-value problem for hyperbolic equations with two independent variables is solved.The solution method proposed by the authors can be used quite widely.

This publication is supported by the grant project 1164 / GF 4 from the Ministry of Education and Science of the Republic of Kazakhstan.

\title{
References
}

1 Whitham G.B. Linear and Nonlinear Waves // John Wiley and Sons. - 1999. - 562 p.

2 Джумабаев Д.С. Признаки однозначной разрешимости линейной краевой задачи для обыкновенных дифференциальных уравнений // Журн. вычисл. мат. и мат. физики. - 1989. - Т. 29. - № 1. C. $50-66$.

\section{Н.Т. Орумбаева, Г. Сабитбекова}

\section{Еркін функциялары бар сызықты емес дифференциалдық теңдеу үшін қойылған бір шеттік есеп туралы}

\begin{abstract}
Мақалада дербес туындылы дифференциалдық теңдеулер үшін жартылай периодты шеттік есеп қарастырылды. Еркін параметрлері бар теңдеулер G.B. Whitham жұмыстарында қарастырылған болатын. Мұндай теңдеулер химиялық технологиялардың және хроматографияның кейбір есептерінде кездеседі. Еркін функциялары бар сызықты емес есепте $u=e^{k z}$ ауыстыруын енгізіп, гиперболалық теңдеулер үшін жартылай периодты сызықты есепті алатын боламыз. Жаңа белгісіз функцияны енгізіп, аралас туынды гиперболалық теңдеулер үшін жартылай периодты сызықты есепті қарапайым дифференциалдық теңдеулер үшін шеттік есептер үйіріне және функционалдық теңдеуге көшеміз. Қарапайым дифференциалдық теңдеулер үшін шеттік есептер үйіріне параметрлеу әдісін қолданып, қарастырып отырған облыста берілген есептің жуық шешімін аламыз. Ұсынылып отырған әдіс мысалмен толықтырылған.
\end{abstract}

Kiлm сөздер: сызықты емес теңдеу, шеттік есеп, дербес туындылы дифференциалдық теңдеу, гиперболалық теңдеу.

\author{
Н.Т. Орумбаева, Г. Сабитбекова
}

\section{О краевой задаче для нелинейного дифференциального уравнения с произвольными функциями}

В статье рассмотрена нелинейная полупериодическая краевая задача для дифференциальных уравнений с частными производными. Уравнения, содержащие произвольные параметры, были изучены в работах G.B. Whithama. Такой вид уравнений встречается в некоторых задачах по химической технологии и хроматографии. Замена $u=e^{k z}$ в нелинейной задаче с произвольными функциями приводит к линейной полупериодической краевой задаче для гиперболических уравнений. Вводя новую неизвестную функцию, линейную полупериодическую краевую задачу для гиперболических уравнений со смешанной производной сведем к семейству краевых задач для обыкновенных дифференциальных 
уравнений и функциональному соотношению. Используя метод параметризации к семейству краевых задач для обыкновенных дифференциальных уравнений, находим приближенные решения данного уравнения в рассматриваемой области. Предложенный метод проиллюстрирован примером.

Ключевые слова: нелинейное уравнение, краевая задача, дифференциальное уравнение в частных производных, гиперболическое уравнение.

\section{References}

1 Whitham G.B. John Wiley and Sons, 1999, 562 p.

2 Dzhumabaev D.S. Computational Mathematics and Mathematical Physics Journal, 1989, 29, 1, p. 50-66. 